\title{
Asystole during pulmonary artery catheter insertion under general anesthesia
}

\author{
Swapna Chaudhuri, Deborah Ortega'1, Kallol Chaudhuri
}

\section{Introduction}

Since the 1970s, flow-guided pulmonary artery (PA) catheters have been employed for the management of critically ill patients, as well as for informed decisionmaking for complex hemodynamic alterations during the perioperative period. Complications associated with their use are well-documented; these include arrhythmias, thrombosis, arterial perforation, and catheter knotting. ${ }^{[1]}$ One of the most common complications is ventricular arrhythmias, which has been reported to be close to $70 \% .{ }^{[2]}$ To the best of our knowledge, this is the first report on the development of asystole during PA catheter insertion under general anesthesia; the incident occurred during its placement, prior to surgical correction of an abdominal aortic aneurysm.

\section{Case Report}

A 63-year-old gentleman was scheduled for repair of a $7 \mathrm{~cm}$ abdominal aortic aneurysm; his past medical history

\section{From:}

Department of Anesthesiology, Texas Tech University Health Sciences

Center, Lubbock, and ${ }^{1}$ Texas Tech University Health Sciences Center,

EI Paso, USA

\section{Correspondence:}

Prof. Swapna Chaudhuri, Department of Anesthesiology, Texas Tech University Health Sciences Center, Lubbock, Texas 79430, USA.

E-mail: Swapna.Chaudhuri@ttuhsc.edu was significant for chronic hypertension and prolonged tobacco abuse. He had undergone femoral-popliteal bypass surgery just three months prior to this admission. His preoperative vital signs were essentially normal and physical examination was unremarkable. A twelve-lead electrocardiogram showed normal sinus rhythm with non-specific ST-T changes; echocardiography performed two days previously revealed normal-sized ventricles, mild anterior hypokinesis, with normal wall motion, and a left ventricular ejection fraction of $55 \%$.

A proposed plan of general endotracheal anesthesia, with invasive hemodynamic monitoring, was discussed with the patient; cell saver services, and four units of packed red cells, were made available for the surgery. After premedication with intravenous midazolam ( $2 \mathrm{mg})$, the patient was transferred to the operating suite; routine monitors revealed a baseline BP of 122 / 97 and normal sinus rhythm in the mid 60s. Following preoxygenation and induction with fentanyl $(3 \mathrm{mcg} / \mathrm{kg}$ ) and etomidate $(0.2 \mathrm{mg} / \mathrm{kg})$, endotracheal intubation was facilitated with cisatracurium $(0.2 \mathrm{mg} / \mathrm{kg})$; general anesthesia was maintained with oxygen : air (50:50), fentanyl, and isoflurane $(0.4-0.8 \%)$.

After left radial artery catheter placement, a 7-Fr percutaneous sheath was introduced via the right 
internal jugular vein. Using waveform analysis, a balloon-tipped, flow-directed PA catheter was then slowly advanced through the sheath. Right atrial tracing, followed by right ventricular waveform, were duly noted until the catheter tip reached $55 \mathrm{~cm}$, when the EKG monitor suddenly demonstrated asystole; systolic BP decreased precipitously from 110 to $<50 \mathrm{mmHg}$, and the arterial waveform disappeared. The PA catheter was immediately deflated and withdrawn completely, without any improvement. Cardiopulmonary resuscitation was initiated with chest compressions and intravenous administration of epinephrine $(1 \mathrm{mg})$ and atropine $(1 \mathrm{mg})$. The patient responded to this management in two to three minutes, with a quick return to sinus tachycardia, with occasional premature ventricular contractions (PVCs) and ST-segment depression. An intraoperative cardiac consultation was obtained; the cardiologist recommended urgent cardiac catheterization and rescheduling of the surgical procedure.

The patient was transferred to the Intensive Care Unit, where he continued to do well, and was extubated later that evening. A coronary angiogram performed the following day showed no new pathology; he underwent open repair of the aortic aneurysm three days later without any further incident.

\section{Discussion}

Despite 40 years of experience with PA catheters, there is ongoing enthusiasm and controversy regarding the benefits and risks associated with this form of invasive monitoring. Even as previous investigations demonstrated its efficacy during goal-directed management in high-risk patients, some recent studies have refuted this argument. ${ }^{[3]}$ Complications can occur during the insertion procedure, when the catheter is in residence, and during removal of the PA catheter.

Dysrhythmias are the primary complications of the catheterization procedure. ${ }^{[4]}$ Premature ventricular and atrial contractions presumably occur from mechanical irritation; these usually resolve spontaneously once the catheter advances through the right heart chambers, and into the pulmonary artery. ${ }^{[2]}$ Sustained ventricular tachycardia or fibrillation is rare, and may be observed in the ischemic myocardium; persistent PVCs or ventricular tachycardia can also indicate catheter kinking, coiling or looping in the right ventricle. ${ }^{[1]}$

Transient right bundle branch block may be seen during catheter insertion; this is postulated to result from the mechanical effects on the conduction system. ${ }^{[1]}$ Additionally, in patients with pre-existing left bundle branch block, a complete heart block has been reported during passage of a PA catheter, as well as with guide wire insertion, during central venous $(\mathrm{CV})$ catheter placement. ${ }^{[5,6]}$ However, there is no previous report of cardiac arrest during PA catheterization in an otherwise normal myocardium.

Complications arising from catheter residence include thrombosis, sepsis, and pulmonary infarction. The most dreaded complication, however, is pulmonary artery rupture; while its incidence is low $(0.03-0.2 \%)$, mortality is estimated to be around $50 \% \cdot{ }^{[4]}$

Finally, complications can occur when the PA catheter is being removed; ventricular arrhythmias have been reported at an incidence similar to insertion. ${ }^{[2]}$ On the other hand, cardiac arrest during PA catheter removal is extremely rare; one case occurred in a 70-year-old male status-post hepatectomy, ${ }^{[7]}$ while another report was during the removal of a CV catheter in a 67-year-old male, following uneventful coronary bypass graft surgery. ${ }^{[8]}$

Given the lack of any new pathological finding, a specific cause for an unexpected cardiac arrest during PA catheter insertion in our patient remained unclear. However, a couple of contributing factors for this suddenonset asystole could be postulated. The depth of the PA catheter (prior to the incident) was observed to be $55 \mathrm{~cm}$, without yet achieving a PA arterial waveform; therefore, it was possible that the catheter was inadvertently coiled in the right ventricle. The mechanical effects of a catheter on the conduction system could cause severe bradycardia and / or complete heart block, ${ }^{[5,6]}$ but it did not provide an explanation for asystole. Another plausible mechanism could be stimulation of ventricular mechanosensitive and chemosensitive receptors triggering a cardioinhibitory reflex..$^{[9]}$ Finally, carotid sinus hypersensitivity, which is well-documented in the elderly, could have played a participatory role in our patient.

Life-threatening complications can occur in approximately $4 \%$ of the patients who undergo PA catheterization. ${ }^{[1]}$ Therefore, perioperative considerations regarding the need for PA catheter monitoring should include patient comorbidity, type of surgery, and skilled practice setting. ${ }^{[4]}$ With the advent of lessinvasive hemodynamic evaluation modalities, such as, transesophageal echocardiography and pulse-wave analysis devices, utility of PA catheters is currently being questioned. ${ }^{[10]}$ Regardless, serious dysrhythmic complications must be kept in mind when choosing a PA catheter for monitoring hemodynamic function and ventricular performance. 


\section{References}

1. Ermakov S, Hoyt JW. Pulmonary artery catheterization. Crit Care Clin 1992;8:773-806.

2. Damen J. Ventricular arrhythmias during insertion and removal of pulmonary artery catheters. Chest 1985;88:190-3.

3. Harvey S, Harrison DA, Singer M, Ashcroft J, Jones CM, Elbourne $\mathrm{D}$, et al. Assessment of the clinical effectiveness of pulmonary artery catheters in management of patients in intensive care (PAC-Man): a randomized controlled trial. Lancet 2005;366:472-7.

4. American Society of Anesthesiologists Task Force on Pulmonary Artery Catheterization. Practice guidelines for pulmonary artery catheterization: an updated report by the American Society of Anesthesiologists Task Force on Pulmonary Artery Catheterization. Anesthesiology 2003;99:988-1014.

5. Abernathy WS. Complete heart block caused by the Swan-Ganz catheter. Chest 1974;65:349.

6. Unnikrishnan D, Idris N, Varshneya N. Complete heart block during central venous catheter placement in a patient with pre-existing left bundle branch block. Br J Anaesth 2003;91:747-9.

7. Nakayama M, Aimono M, Kawana S, Oomori H, Watanabe H, Namiki A. Cardiac arrest during removal of a pulmonary artery catheter. Can J Anaesth 1996;43:972-4.

8. Goel S, Dhir A. Cardiac arrest after the removal of a central venous catheter. J Cardiothorac Vasc Anesth 2008;22:174-5.

9. Kinsella SM, Tuckey JP. Perioperative bradycardia and asystole: relationship to vasovagal syncope and the Bezold-Jarisch reflex. Br J Anaesth 2001;86:859-68.

10. Hadian M, Pinsky MR. Evidence-based review of the use of the pulmonary artery catheter: impact data and complications. Crit Care 2006;10 Suppl 3:S8.

How to cite this article: Chaudhuri S, Ortega D, Chaudhuri K. Asystole during pulmonary artery catheter insertion under general anesthesia. Indian J Crit Care Med 2012;16:41-3.

Source of Support: Nil, Conflict of Interest: None declared.

\section{Dispatch and return notification by E-mail}

The journal now sends email notification to its members on dispatch of a print issue. The notification is sent to those members who have provided their email address to the association/journal office. The email alerts you about an outdated address and return of issue due to incomplete/incorrect address.

If you wish to receive such email notification, please send your email along with the membership number and full mailing address to the editorial office by email. 